\title{
Should surgeons control fluoroscopy during urology procedures?
}

\author{
Jeremy Setterfield, MD; James Watterson, MD; ${ }^{-1}$ Mathew Playfair, MD:2 Luke T. Lavallée, MD, ${ }^{1,3}$ \\ Matthew Roberts, MD;' Brian Blew, MD; J. Stuart Oake, MD'
}

'Department of Urology, The Ottawa Hospital, Ottawa, ON, Canada; ${ }^{2}$ College of Medical and Dental Sciences, University of Birmingham, Birmingham, United Kingdom; ${ }^{3}$ Ottawa Hospital Research Institute, Ottawa, ON, Canada

Cite as: Can Urol Assoc J 2016;10(11-12):398-402. http://dx.doi.org/10.5489/cuaj.3895

See related commentary on page 403.

\section{Abstract}

Introduction: Our study explored the impact of switching from surgeon- to radiation technologist (RT)-controlled fluoroscopy on fluoroscopy and operative times. We also identified factors impacting fluoroscopy and operative times for ureteroscopy (URS) with laser lithotripsy.

Methods: Patients undergoing urological procedures requiring fluoroscopy six months before and after the change from surgeonto RT-controlled fluoroscopy were identified. Median fluoroscopy and operative times were compared between cohorts. Subgroup analyses were performed based on procedure performed. A multivariate analysis identified factors associated with increased fluoroscopy and operative times for URS with laser lithotripsy.

Results: Overall, no difference was found between surgeon and RT cohorts for fluoroscopy (58.0 vs. 56.7 seconds; $p=0.34$ ) or operative times (39 vs. 36 minutes; $p=0.14$ ). For URS with laser lithotripsy, fluoroscopy and operative times were longer in the surgeon-controlled cohort (76.0 vs. 54.0 seconds; $\mathrm{p}<0.01$ and 48 vs. 40 minutes; $p<0.01$, respectively). For URS only, fluoroscopy time was decreased in the surgeon-controlled cohort (47.0 vs. 73.0 seconds; $p=0.01$ ). For URS with laser lithotripsy, factors independently associated with increased fluoroscopy time were male sex, flexible URS, glidewire use, and difficult ureteric stent insertion $(p<0.05)$. Flexible ureteroscopy, glidewire use, previous ureteric stent placement, and difficult ureteric stent insertion were independently associated with increased operative time $(p<0.05)$. Conclusions: Fluoroscopy and operative times are not significantly influenced by who controls fluoroscopy during urologic procedures. Patients undergoing URS with laser lithotripsy have decreased fluoroscopy and operative times with RT-controlled fluoroscopy. Patients undergoing URS only have decreased fluoroscopy times with surgeon-controlled fluoroscopy.

\section{Introduction}

The use of ionizing radiation for diagnostic and therapeutic endeavours has become ubiquitous in the field of modern medicine. In the recent past, population-based studies have been undertaken in an attempt to quantify the risk of ionizing radiation exposure to patients. Of particular concern is the potential for secondary malignancies.

A comprehensive review of biological and epidemiological data related to ionizing radiation was published by the National Academy of Sciences' National Research Council in 2006. ${ }^{1}$ Small doses of ionizing radiation, similar to those encountered with computed tomography (CT) imaging, were associated with an increased risk of cancer. Although quantifying the exact risk to a patient is difficult, there is universal agreement that limiting patient exposure to ionizing radiation is of paramount importance. The principle of ALARA (as low as reasonably achievable) is supported by all regulatory bodies governing the use of ionizing radiation. ${ }^{2}$

Patients with nephrolithiasis are at a high risk for exposure to ionizing radiation. The high availability and sensitivity/specificity of CT imaging has made it the most used investigation for renal colic. ${ }^{3}$ Therapeutic intervention with both extracorporeal shockwave lithotripsy and ureteroscopy (URS) with laser lithotripsy involve ionizing radiation exposure through the use of fluoroscopy. Patients with nephrolithiasis have a $30-40 \%$ recurrence rate, making the chance of repeat investigation and treatment significant. ${ }^{4}$

Limiting fluoroscopy can decrease radiation exposure to patients during urological procedures. Previous studies evaluating methods to decrease fluoroscopy time are encouraging. One study demonstrated an $82 \%$ reduction in fluoroscopy time with the use of a dedicated protocol, including a laserguided C-arm, a designated fluoroscopy technician, and substituting visual clues for fluoroscopic vision. ${ }^{5}$ Another study has shown that providing surgeons with feedback on their fluoroscopic time can decrease fluoroscopy use. ${ }^{6}$ 
In an effort to decrease fluoroscopy exposure, the control of fluoroscopy during urological procedures at our institution was changed from surgeon-controlled via use of a foot pedal to manually controlled by the radiation technologist (RT). This change was implemented for all endourological procedures with the exception of percutaneous nephrolithotomy. Our study was designed to determine the impact of this change on fluoroscopy and operative times. Our hypothesis was that the change in fluoroscopy control would have no effect on fluoroscopy and operative times. We also aimed to determine patient and surgical factors impacting fluoroscopy and operative times for patients undergoing URS with laser lithotripsy.

\section{Methods}

This study was approved by the Ottawa Health Science Network Research Ethics Board.

Patients who underwent urological procedures requiring fluoroscopy between July 1, 2013 and July 31, 2014 at The Ottawa General Hospital were included. Patients undergoing percutaneous nephrolithotomy were not included, as the decision was made to continue with surgeon-controlled fluoroscopy for this operation. Two cohorts of patients were defined: patients who had their operation performed with surgeon-controlled fluoroscopy (between July 1,2013 and January 19, 2014) and patients who had their operation performed with RT-controlled fluoroscopy (between January 20, 2014 and July 31, 2014).

Patient characteristics were extracted from our hospital's electronic medical record. Fluoroscopy time was retrieved from the radiology report dictated by the radiologist reviewing the fluoroscopy images. When not included in the dictation, fluoroscopy time was obtained from our electronic imaging system. Information regarding operating room time was extracted from the operative record, where it is reported as a mandatory field by nursing staff. Relevant operative details, including procedure performed and equipment used, were obtained from the operative notes. Information on stone number, size, and location, was obtained from preoperative imaging when not included in the operative dictation.

Fluoroscopy time and operative time were defined as our primary and secondary endpoints, respectively. A Wilcoxon two-sample test was performed to compare median fluoroscopy and operative time between groups. A subgroup analysis was done between the two cohorts by stratifying patients by procedure performed.

We identified patient and surgical factors associated with fluoroscopy and operative times for patients who underwent URS with laser lithotripsy using a multivariate Cox proportional hazard model. The primary outcome was time to cessation of fluoroscopy. The secondary outcome was time to completion of the operation. Variables analyzed included study cohort, sex, type of ureteroscope used, use of a hydrophilic glidewire, use of a nitinol basket, previous ureteric stent placement, and stone factors (such as size, location, number, and presence of impaction). Analyses were performed using SAS version 9.3. Statistical significance was defined at a $p$ value $<0.05$.

\section{Results}

We identified 436 study participants. Demographic and surgical information is presented in Table 1 .

Overall, there was no significant difference between the median fluoroscopy and operative time between cohorts (Fig. 1). Median fluoroscopy time was 58.0 seconds (interquartile range [IQR] 33.0-108.0 seconds) in the surgeon-controlled fluoroscopy cohort and 56.7 seconds (IQR 26.0-98.0 seconds) in the RT-controlled fluoroscopy cohort ( $p=0.34)$. Median operative time was 39 minutes (IQR 25-58 minutes) in the surgeon-controlled cohort vs. 36 minutes (IQR 24-49 minutes) in the RT cohort ( $p=0.14)$.

A subgroup analysis was performed for both fluoroscopy and operative time by stratifying study participants by procedure performed (Figs. 2, 3). In patients undergoing URS with laser lithotripsy, there was a significant difference in median fluoroscopy and operative time between cohorts. Patients in the surgeon-controlled cohort had a significantly longer median fluoroscopy time: 76.0 seconds (IQR 41.0-129.0 seconds) compared to 54.0 seconds (IQR 28.0-98.4 seconds) in the RT cohort ( $\mathrm{p}<0.01)$. Patients in the surgeon-controlled cohort also had a significantly longer median operative time: 48 minutes (IQR 36-66 minutes)

\begin{tabular}{lcc}
\hline Table 1. Demographic and surgical information & $\begin{array}{c}\text { Surgeon- } \\
\text { controlled } \\
\text { fluoroscopy } \\
\text { cohort (n=206) }\end{array}$ & $\begin{array}{c}\text { Radiation } \\
\text { technologist- } \\
\text { controlled } \\
\text { fluooscopy cohort } \\
\text { (n=230) }\end{array}$ \\
\hline Sex & $111(53.9 \%)$ & $129(56.1 \%)$ \\
Male & $95(46.1 \%)$ & $101(43.9 \%)$ \\
Female & $111(53.9 \%)$ & $126(54.8 \%)$ \\
Procedure performed & $12(5.8 \%)$ & $12(5.2 \%)$ \\
URS with laser lithotripsy & $32(15.5 \%)$ & $37(16.1 \%)$ \\
URS with biopsy & $48(23.3 \%)$ & $51(22.2 \%)$ \\
URS only & $3(1.5 \%)$ & $4(1.7 \%)$ \\
Ureteric stent insertion & $25(12.1 \%)$ & $41(17.8 \%)$ \\
Retrograde pyelogram & & \\
Previously stented & $48(23.3 \%)$ & $56(24.4 \%)$ \\
Type of ureteroscope used & $57(27.7 \%)$ & $67(29.1 \%)$ \\
None & $70(34.0 \%)$ & $74(32.2 \%)$ \\
Semi-rigid & $31(15.1 \%)$ & $33(14.4 \%)$ \\
Flexible & $174(84.5 \%)$ & $207(90.0 \%)$ \\
Both & & \\
Ureteric stent left & & \\
\hline URS: ureteroscopy. & & \\
\hline
\end{tabular}




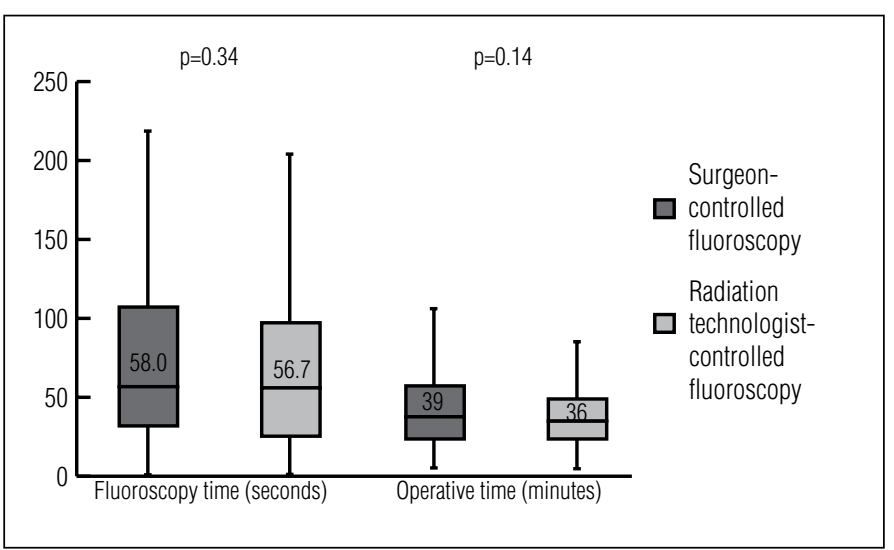

Fig. 1. Box and whisker plot comparing fluoroscopy and operative times between study cohorts. Boxes indicate the lower quartile, median, and upper quartile values. Whiskers denote the minimum and maximum values excluding outliers.

compared to 40 minutes (IQR 30-49 minutes) in the RT cohort $(p<0.01)$.

For patients undergoing URS alone, median fluoroscopy time was significantly shorter in the surgeon-controlled cohort: 47.0 seconds (IQR 12.5-78.7 seconds) compared to 73.0 seconds (IQR 32.0-146.0 seconds) in the RT cohort $(p=0.01)$. There was no significant difference in fluoroscopy or operative time between cohorts for patients undergoing URS with biopsy, ureteric stent insertion, or retrograde pyelogram.

A multivariate analysis was completed for patients undergoing ureteroscopy with laser lithotripsy. Factors independently associated with increased fluoroscopy time were male sex, flexible URS, use of a hydrophilic glidewire, and difficulty with ureteric stent insertion $(p<0.05)$. Previous ureteric stent placement was independently associated with decreased fluoroscopy time ( $p=0.03$ ) (Fig. 4).

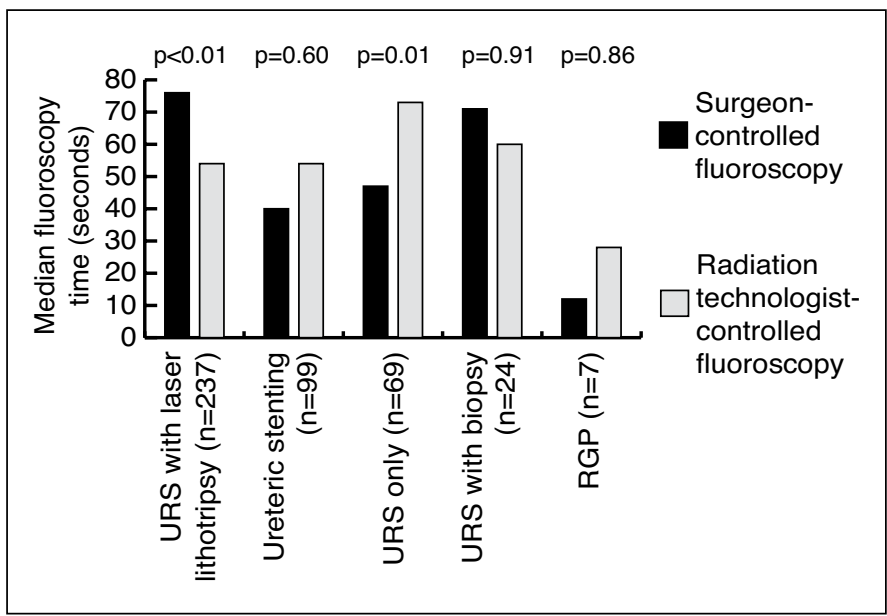

Fig. 2. Median fluoroscopy times stratified by study cohort and procedure performed. Cohorts were compared using a Wilcoxon two-sample test. RGP: retrograde pyelogram; URS: ureteroscopy.
Factors independently associated with increased operative time were flexible URS, use of a hydrophilic glidewire, previous ureteric stent placement, and difficulty with ureteric stent insertion $(\mathrm{p}<0.05)$. RT-controlled fluoroscopy was independently associated with decreased operative time $(\mathrm{p}=0.02)$ (Fig. 5).

\section{Discussion}

Our study demonstrates that, overall, control of fluoroscopy by the surgeon or by a RT does not significantly influence fluoroscopy time or operative time for endourological procedures requiring fluoroscopy.

Our subgroup analysis yielded several interesting results. In patients undergoing URS with laser lithotripsy (representing over $50 \%$ of study participants), there was a significant difference in fluoroscopy and operative times between cohorts. The RT-controlled cohort demonstrated significantly less fluoroscopy exposure (54.0 vs. 76.0 seconds) and shorter operative times (40 vs. 48 minutes). This data would suggest that allowing the RT to control fluoroscopy for these cases may be advantageous. It is important to note that our multivariate analysis for patients undergoing URS with laser lithotripsy did not find RT-controlled fluoroscopy to be independently associated with decreased fluoroscopy time, suggesting that differences in patient and other surgical factors between cohorts may account for the discrepancy between median fluoroscopy times seen in this subgroup. However, our multivariate analysis for operative time did conclude that RT-controlled fluoroscopy was independently associated with decreased operative times. A possible explanation is the challenge of efficiently operating two different devices (the holmium laser and the fluoroscopic unit) with foot pedals during the operation.

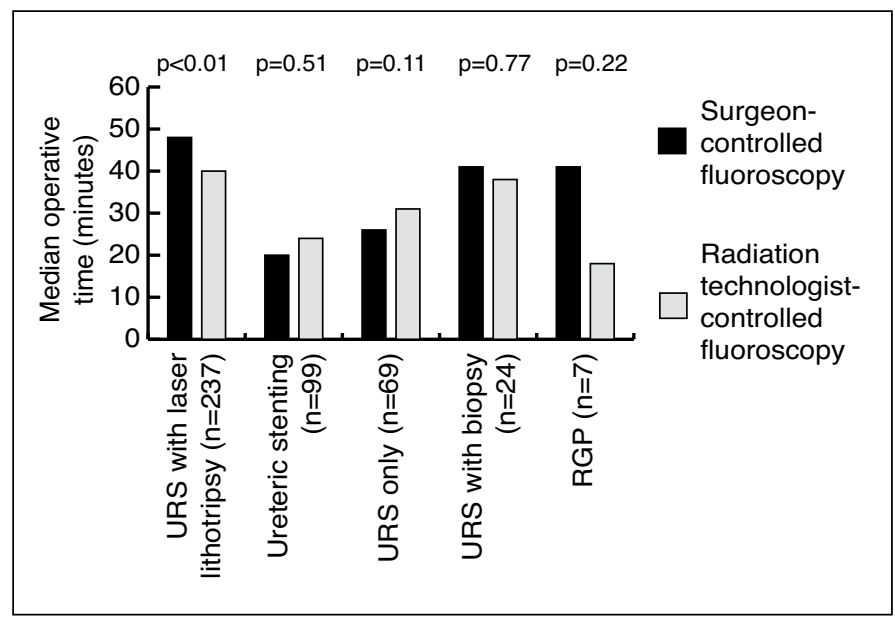

Fig. 3. Median operative times stratified by study cohort and procedure performed. Cohorts were compared using a Wilcoxon two-sample test. RGP: retrograde pyelogram; URS: ureteroscopy. 


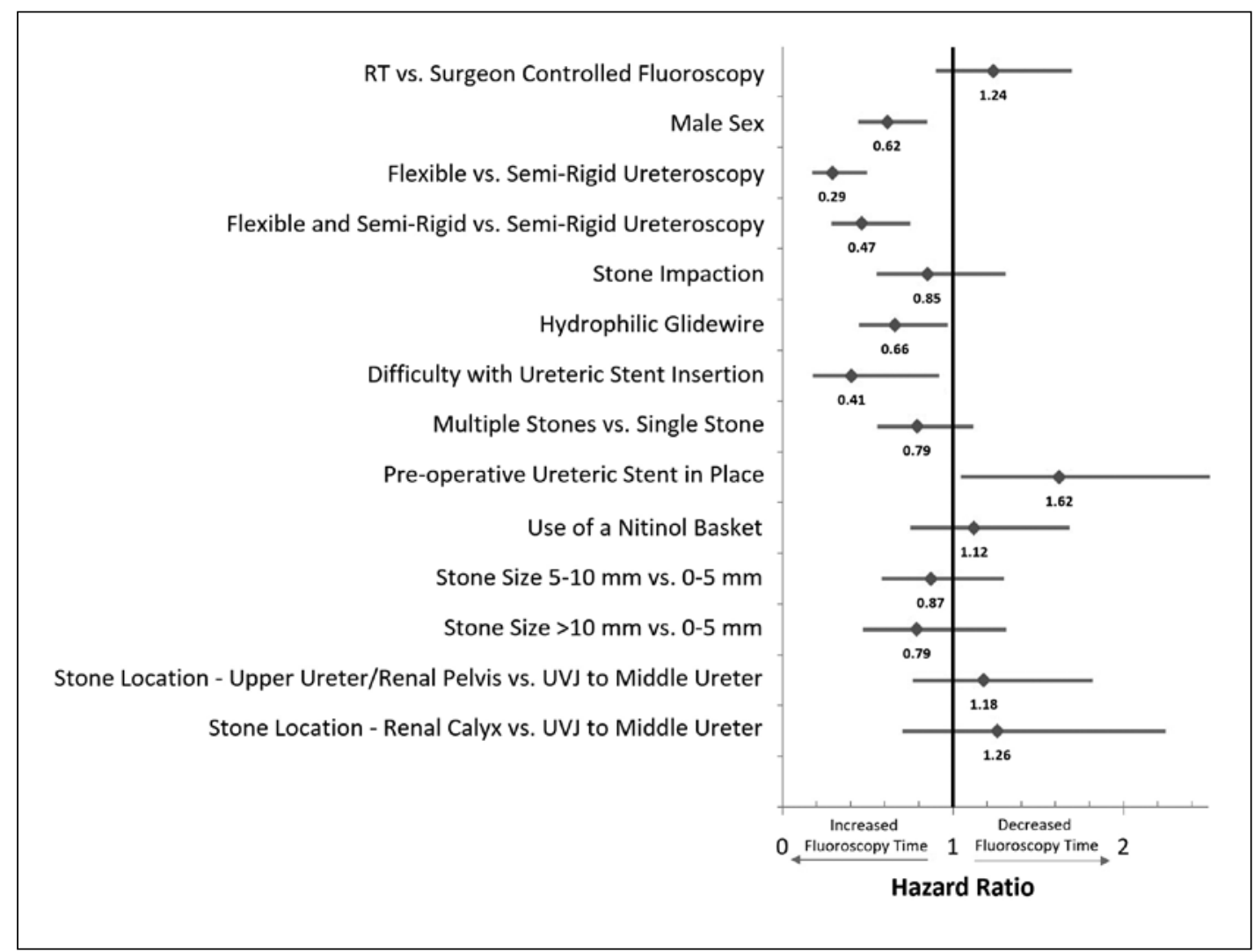

Fig. 4. Forest plot illustrating the effect of patient and surgical factors on cessation of fluoroscopy exposure. Hazard ratios represent the influence of a given factor on the chance of terminating fluoroscopy at any given time point during the operation. Horizontal bars indicate $95 \%$ confidence intervals.

For patients undergoing URS only, surgeon-controlled fluoroscopy was associated with a significant decrease in fluoroscopy time (47.0 vs. 73.0 seconds). Indications for URS alone included suspected ureteric or renal calculi, suspected upper tract urothelial cancer, and ureteral trauma/ stricture disease. It is conceivable that RTs would be less sure of the optimal time for fluoroscopy exposure in these more nuanced cases. With only a single pedal to operate, surgeon-controlled fluoroscopy may be more precise, as it removes the need for communication with the RT for fluoroscopic activation. A surgeon-controlled approach may be more appropriate for these cases.

In patients undergoing URS with laser lithotripsy, factors predictive of a significantly increased fluoroscopy time included male sex, flexible URS, use of a hydrophilic glidewire, and difficulty with ureteric stent insertion. Our results are in agreement with previous studies that have shown that male sex, ureteric stent insertion, and flexible URS increases fluoroscopy time during URS.7,8

Factors predictive of a significantly increased operative time included flexible URS, use of a hydrophilic glidewire, previous ureteric stent placement, and difficulty with ureteric stent insertion. Use of a flexible ureteroscope takes longer, given the need for placement of a second guidewire or inser- tion of a ureteral access sheath. Navigation with a flexible scope can also be more challenging and take longer than when using a semi-rigid ureteroscope.

Our study does have several limitations that must be considered when interpreting the results. Firstly, our study was performed retrospectively through analysis of electronic medical records and imaging reports. The electronic records are assumed to be accurate, but there is no way to verify this. Secondly, our study is not randomized. There may be differences between the surgeon-controlled and RT-controlled cohorts despite attempts to include all relevant demographic and surgical data points. Given the retrospective nature of our study, we were not able to determine whether surgeoncontrolled fluoroscopy had been controlled by a staff physician or a urology resident. It is likely that experience with fluoroscopy control and urological procedures has an influence on fluoroscopy and operative times.

Despite these limitations, our study is the first of its kind to look at whether fluoroscopy and operative times are dependent on the individual controlling fluoroscopic exposure. Our study includes a large contemporary cohort of patients undergoing routine endourological procedures. Given the limited exclusion criteria, our results should be applicable to most patients presenting for urological care. 
Setterfield et al.

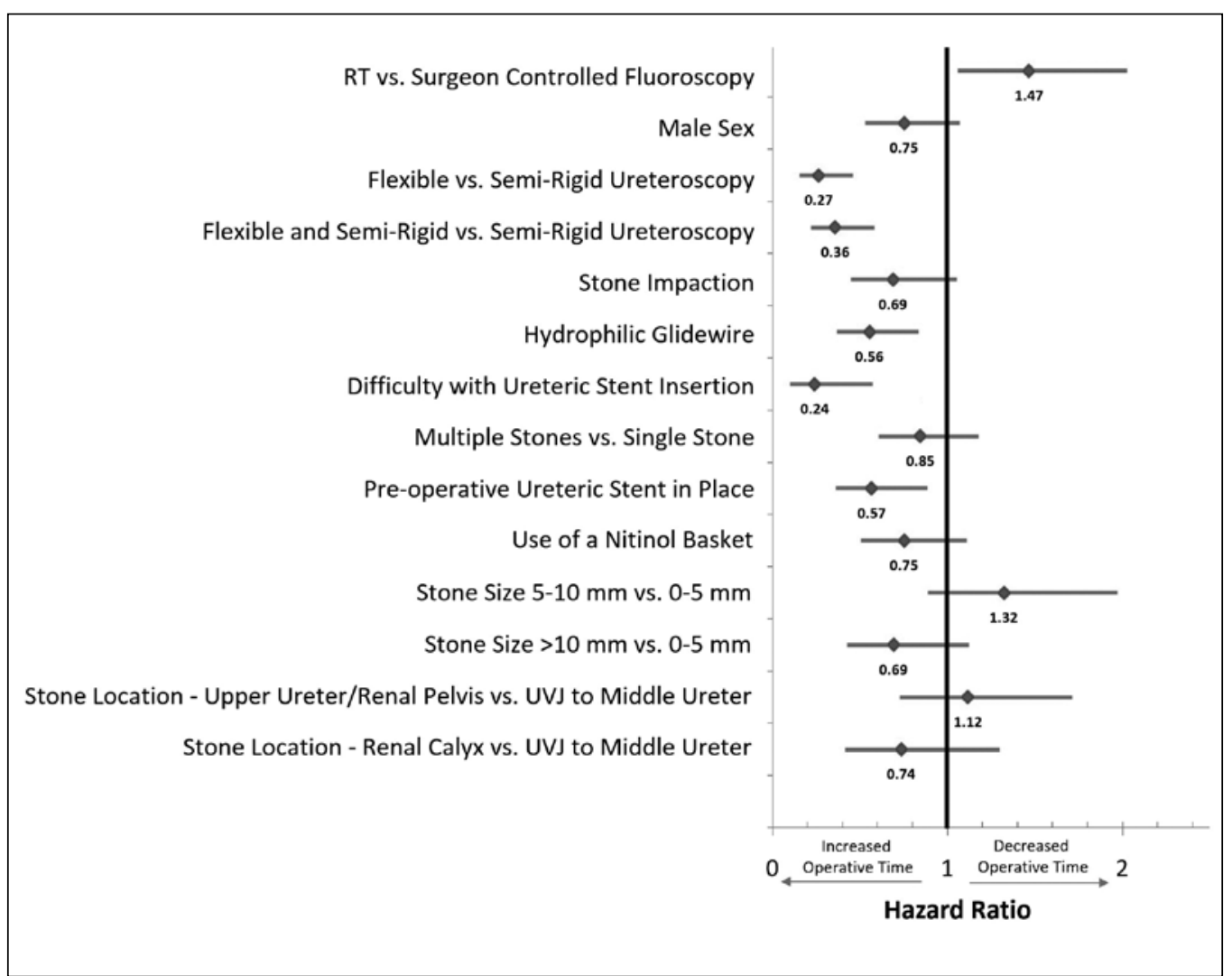

Fig. 5. Forest plot illustrating the effect of patient and surgical factors on completion of the operation. Hazard ratios represent the influence of a given factor on the chance of completing the procedure at any given time point during the operation. Horizontal bars indicate $95 \%$ confidence intervals.

Future research possibilities would include the completion of a randomized, prospective trial. A randomized, prospective pediatric trial at the Children's Hospital in Boston using similar outcome measures is currently accruing patients and the results from this trial may help provide further guidance as to the optimal use of fluoroscopy in urology procedures. ${ }^{9}$

Competing interests: Dr. Lavallée has been an advisor for Ferring and Sanofi, and has received a research grant from Sanofi. Dr. Roberts is a member of a Speaker's Bureau for Abbott. Dr. Oake has received speaking fees for Pfizer. The remaining authors report no competing personal or financial interests.

This paper has been peer-reviewed.

\section{References}

1. National Research Council of the National Academies. Health risks from exposure to low levels of ionizing radiation. BEIR VII Phase 2. The National Academies Press 2006, Washington, 2006.

2. Canadian Nuclear Safety Commission. Keeping radiation exposures and doses "as low as reasonably achievable (ALARA)." Regulatory Guide G-129, Revision 1; October 2004. http://nuclearsafety.gc.ca/ pubs_catalogue/uploads/G129revl_e.pdf. Accessed October 24, 2016.

3. Portis AJ, Sundaram CP. Diagnosis and initial management of kidney stones. Am Fam Physician 2001;63:1329-40.

4. Strohmaier WL. Course of calcium stone disease without treatment. What can we expect? Eur Urol 2000;37:339-44. http://dx.doi.org/10.1159/000052367

5. Greene DJ, Tenggadjaja CF, Bowman RJ, et al. Comparison of a reduced radiation fluoroscopy protocol to conventional fluoroscopy during uncomplicated ureteroscopy. Urology 2011;78:286-90. http://dx.doi. org/10.1016/i.urology.2010.11.020

6. $\mathrm{Ngo} \mathrm{TC}$, Macleod LC, Rosenstein DI, et al. Tracking intraoperative fluoroscopy utilization reduces radiation exposure during ureteroscopy. J Endourol 2011;25:763-7. http://dx.doi.org/10.1089/end.2010.0624

7. Violette PD, Szymanski KM, Anidjar M, et al. Factors determining fluoroscopy time during ureteroscopy. J Endourol 2011;25:1837-40. http://dx.doi.org/10.1089/end.2011.0204

8. Weld LR, Nwoye U0, Knight RB, et al. Fluoroscopy time during uncomplicated unilateral ureteroscopy for urolithiasis decreases with urology resident experience. World J Urol 2015;33:119-24. http://dx.doi. org/10.1007/s00345-014-1264-y

9. Clinicaltrials.gov. Assessment of fluoroscopy times with surgeon vs. technologist control. Updated January 26, 2016. htrps://clinicaltrials.gov/show/NCT02224287. Accessed February 17, 2016.

Correspondence: Dr. Jeremy Setterfield, Department of Urology, The Ottawa Hospital, Ottawa, ON, Canada; jeremy.setterfield@gmail.com 